\title{
Effect of astaxanthin on ethylene glycol induced nephrolithiasis
}

\author{
Manju Alex ${ }^{\mathrm{a}}$, Mathews V Varghese ${ }^{\mathrm{a}}$, M Abhilash ${ }^{\mathrm{a}}, \mathrm{M}$ V Sauganth Paul ${ }^{\mathrm{a}}$ \\ R Harikumaran Nair ${ }^{\mathrm{a}}$ \\ ${ }^{a}$ School of Biosciences, Mahatma Gandhi University, Priyadarsini Hills P.O. Kottayam, Kerala, India, 686560
}

\begin{abstract}
Nephrolithiasis is one of the most common and painful of urological disorders with a high prevalence rate. The role of calcium oxalate crystals, which are the predominant component of kidney stones in generating oxidative stress, have been clearly demonstrated in previous studies. Astaxanthin, found in marine organisms is a dietary xanthophyll carotenoid with enhanced antioxidative properties and pharmacological effects. In the present study, we have investigated the effect of this natural antioxidant, at a daily dose of $25 \mathrm{mg} / \mathrm{kg}$ in experimental calcium oxalate nephrolithiasis in male Wistar rats. Liver function markers, hepatic antioxidants, albumin creatinine ratios, renal calcium content and changes in body and kidney weight have been studied to evaluate the effect of this carotenoid in vivo. The effect of citrate, a component of most pharmaceutical drugs for management of nephrolithiasis has also been evaluated for the purpose of comparison with astaxanthin treatment. Astaxanthin is seen to exert a protective effect on the liver and kidney tissues in ethylene glycol treated rats by improving the liver function, restoring the activity of the hepatic antioxidant enzymes, decreasing the albumin creatinine ratios and calcium levels and maintaining the organ to body weight ratio. Our results also indicate that astaxanthin administration is more beneficial than citrate treatment.
\end{abstract}

Keywords: astaxanthin, calcium oxalate, citrate, nephrolithiasis, nephroprotective.

\section{Introduction}

Kidney stone disease is one of the most painful and common urological disorders affecting mankind since centuries. Calcium containing stones especially calcium oxy monohydrate (COM) are the most commonly occurring ones to the extent of 75-90\% [1]. Several invasive and non invasive procedures are currently employed for the treatment of kidney stones but they are limited by their high cost, severe adverse effects and high recurrence rates [2]. Hence researchers in this area are confronted with the need to develop natural remedies to combat this disease. Organ weight can be the most sensitive indicator of the effect of an experimental compound. Significant differences may occur between the organ weights of a treated and non treated animal even in the absence of evident morphological changes. A criterion called relative organ weight is used here which accommodates the changes in body weights of the experimental animals [3]. Liver is the target organ for the metabolism of all drugs and toxic chemicals. Any drug administered will exert its action at the hepatic level. Hence an evaluation of the hepatic function has been carried out in this study as well by estimating the alanine aminotransferase (ALT), aspartate aminotransferase (AST) and alkaline phosphatase levels (ALP) as they are released into blood following hepatocellular damage [4]. Kidney is vital in maintenance of homeostasis through the excretion of catabolites like urea, creatinine, uric acid and elevated concentration of these indicates compromised renal function. Oxidative stress has been implicated as a mechanism in oxalate induced renal damage. Hence treatment with antioxidants could be a possible strategy to effectively reduce the damage caused by COM stones. Astaxanthin (3, 3-dihydroxybeta, beta-carotene-4, 4-dione, CAS 472-61-7) is a naturally occurring carotenoid found in variety of plants, algae and sea food such as salmon, trout, red sea bream, shrimp, lobster and fish eggs. It is also present in birds such as flamingos and quails. In many of the aquatic animals in which it is found, astaxanthin has several essential biological functions including protection against oxidation of essential polyunsaturated fatty acids; protection against UV light effects; immune response; pigmentation; etc [5]. Some microorganisms are rich in astaxanthin - the chlorophyte alga Haematococcus pluvialis is believed to accumulate the highest levels of astaxanthin in nature. Astaxanthin exhibits 100-500 times more antioxidant property than $\alpha$-tocopherol [6]. Moreover, astaxanthin has been found to provide many essential biological functions, including protection against lipid-membrane peroxidation of essential polyunsaturated fatty acids and proteins, DNA damage and UV light effects; it also plays an important role in immunological defence [7]. This has stirred great interest in astaxanthin and promoted numerous research studies concerning its potential benefits to humans and animals.

The current study was undertaken to assess the effect of astaxanthin at a dose of $25 \mathrm{mg} / \mathrm{kg}$ body weight on relative organ weights, liver marker enzymes, hepatic antioxidant enzymes, calcium levels and serum and urine albumin creatinine ratios in experimentally induced calcium oxalate stone disease. A comparison has also been made with citrate administration used in pharmaceutical preparations for the clinical treatment of kidney stones. 


\subsection{Reagents and Chemicals}

\section{Material and methods}

Astaxanthin, $1.5 \%$ phytoextract powder derived from Haematococcus microalgae was used for the study. It was dissolved in distilled water and $2 \mathrm{ml}$ is fed to each rat such that they receive a dose equivalent to $25 \mathrm{mg} / \mathrm{kg}$. Ethylene glycol, 24, 25-Dihydroxycholecalciferol (Vitamin $\mathrm{D}_{3}$ ) and Potassium citrate were obtained from Merck India Ltd. All other chemicals were of purest analytical grade.

\subsection{Animals}

Thirty male wistar rats of 220-250g were purchased from Small Animal Breeding Station (SABS) of Govt. Veterinary College, Mannuthy, Thrissur, Kerala, India, divided into five groups of six rats each and allowed to acclimatise for a period of seven days. Animal care and treatment were in conformity with the guidelines of Institutional Animal Ethical Committee, School of Biosciences, Mahatma Gandhi University, Kerala, India. All animals were housed in a constant temperature room $\left(25 \pm 2^{\circ} \mathrm{C}\right)$ with $12 \mathrm{~h}$ light / dark cycle, fed standard diet (Amrut Rat Pellet, Pranav Agro Industries Ltd, Pune, India) and given water ad libitum throughout the experimental period. The animals were grouped as follows ; Group I-Control(fed on laboratory rat chow), Group II-Astaxanthin control (Astaxanthin 25mg/kg body wt. for 21 days), Group III-Calculi induced (0.75\% Ethylene glycol daily and $0.5 \mu \mathrm{g}$ Vitamin $\mathrm{D}_{3}$ every other day for 28 days, Group IV-Astaxanthin treated (Astaxanthin $25 \mathrm{mg} / \mathrm{kg}$ body wt. for 21 days following calculi induction as in group III) and Group V-Citrate treated (Potassium citrate at $100 \mathrm{mg} / \mathrm{kg}$ body wt. for 21 days after calculi induction as in group III). All the rats were fed by oral intubation. Their body weights were recorded periodically.

On the $21^{\text {st }}$ day all the animals were weighed, kept in metabolic cages individually and their urine was collected. A drop of concentrated hydrochloric acid was added to the urine before being stored at $4^{\circ} \mathrm{C}$. All the rats were then euthanized, decapitated and blood was collected. Both the kidneys were excised, washed in ice cold $0.15 \mathrm{M}$ saline, blotted on a tissue paper and weighed and then used for flame photometry.

\subsection{Kidney weights and Renoprotective index}

Relative kidney weight was calculated dividing the absolute weight of the kidney by weight of the animal on the $21^{\text {st }}$ day. The kidney protecting activity of astaxanthin was expressed as renoprotective index which was calculated using the formula

"R $=[1-(\mathrm{X}-\mathrm{C}) /(\mathrm{T}-\mathrm{C})] \mathrm{x} 100 "$

$\mathrm{X}$ is the mean value of astaxanthin or citrate treated group

$\mathrm{T}$ is the mean value of calculi induced group and $\mathrm{C}$ is the mean value of control group [8].

\subsection{Biochemical assays}

Serum was separated by centrifugation at $10,000 \mathrm{rpm}$ for $10 \mathrm{~min}$ and analyzed for creatinine, albumin and enzymes AST, ALT and ALP. Urine was analyzed for albumin and creatinine content. All estimations were carried out with the kits provided by Span Diagnostics Ltd. as per manufacture's instructions.

\subsection{Serum and Urine albumin/creatinine ratio (ACR)}

The albumin to creatinine ratio was calculated by dividing the urinary albumin value in $\mathrm{mg} / \mathrm{dl}$ by creatinine value in $\mathrm{mg} / \mathrm{dl}$.

\subsection{Hepatic antioxidants}

Lipid peroxidation in the liver was measured by thiobarbituric acid (TBARS) method of Beuge and Aust (1978) [9]. A principle component of TBARS is malondialdehyde (MDA) a product of lipid peroxidation. Superoxide dismutase (SOD) was measured by the method of Kakkar et al., (1984) [10] Catalase (CAT) by Aebi (1974) [11] Glutathione peroxidase (GPx) by Rotruck et al., (1973) [12] Glutathione-S- transferase (GST) by Habig (1974) [13] and the protein content by Lowry et al., (1951) [14].

\subsection{Flame photometry}

The kidneys were dried in an oven at $100^{\circ} \mathrm{C}$ ashed at $600{ }^{\circ} \mathrm{C}$ and digested in $1: 1 \mathrm{HCl}$ until the liquid became transparent. After calibration using a standard calcium solution, the calcium content of the tissue was measured by flame photometry (BWB XP Flame Photometer). The results are expressed as parts per million.

\subsection{Statistical analysis}

The results were analyzed using a statistical programme SPSS/PC+, version 10 (SPSS Inc. Chicago, IL, USA). One way ANOVA was employed for comparison among the three groups. LSD post hoc multiple comparison test was used to determine significant difference among groups. $\mathrm{p}<0.05$ was considered significant. 


\section{Results}

3.1 Body weights and Kidney to body weight ratio

The body weights of all animals were more or less similar at the beginning of the experiment. A progressive decrease in the body weights was noted in the calculi induced group till the end of the experimental period. The astaxanthin treated groups however showed an increase in body weights similar to the controls. The citrate group showed only a nominal increase in the body weight (Table 1). The kidneys excised from the calculi induced group were larger and heavier than the other groups. The absolute kidney weight was found to be significantly higher in the calculi induced group where as all other groups show similar values (Table 2). This could be due to the deposition of COM crystals in the kidneys of stone forming animals. The relative kidney weights also followed the same pattern with a significant lowering of the values for the Astaxanthin treated group as compared to citrate treatment. Also, the renoprotective percentage of astaxanthin was higher $(72.1 \%)$ as compared to citrate treatment $(51.4 \%)$.

\subsection{Serum enzymes}

The serum AST, ALT and ALP values were significantly increased in the calculi induced group when compared to the controls. Astaxanthin treatment significantly brought down the values to normal levels $(\mathrm{p}<0.05)$. Citrate administration did not bring about a significant decrease in all the three parameters (Table 3 ).

\subsection{Hepatic antioxidant status}

The lipid peroxidation was significantly increased in the liver of the calculi induced group where as it reduced significantly in the astaxanthin treated group $(\mathrm{p}<0.05)$. There was only a slight reduction in the citrate treated group which was however not significant. The activities of the enzymes SOD, CAT, GPx and GST were attenuated in the calculi group. The activities of these enzymes were restored to the normal level by the administration with astaxanthin. Citrate treatment failed to bring back the levels of these antioxidant enzymes to normalcy (Table 4).

\subsection{ACR ratios}

Treatment with astaxanthin brought down the elevated value of both serum and urinary ACR ratios whereas citrate administration caused only a slight decrease. (Fig.1and 2)

\subsection{Flame photometry}

The results of the flame photometry (Fig. 3) indicated that in the calculi induced group (Group III), calcium content was very high obviously due to the deposition of calcium oxalate crystals. The control groups (Group I, II) showed less calcium content possibly due to presence of intracellular calcium. The astaxanthin and citrate treated groups (Group IV, V) showed reduction in calcium levels which was higher than the controls but significantly lower than the calculi induced group.

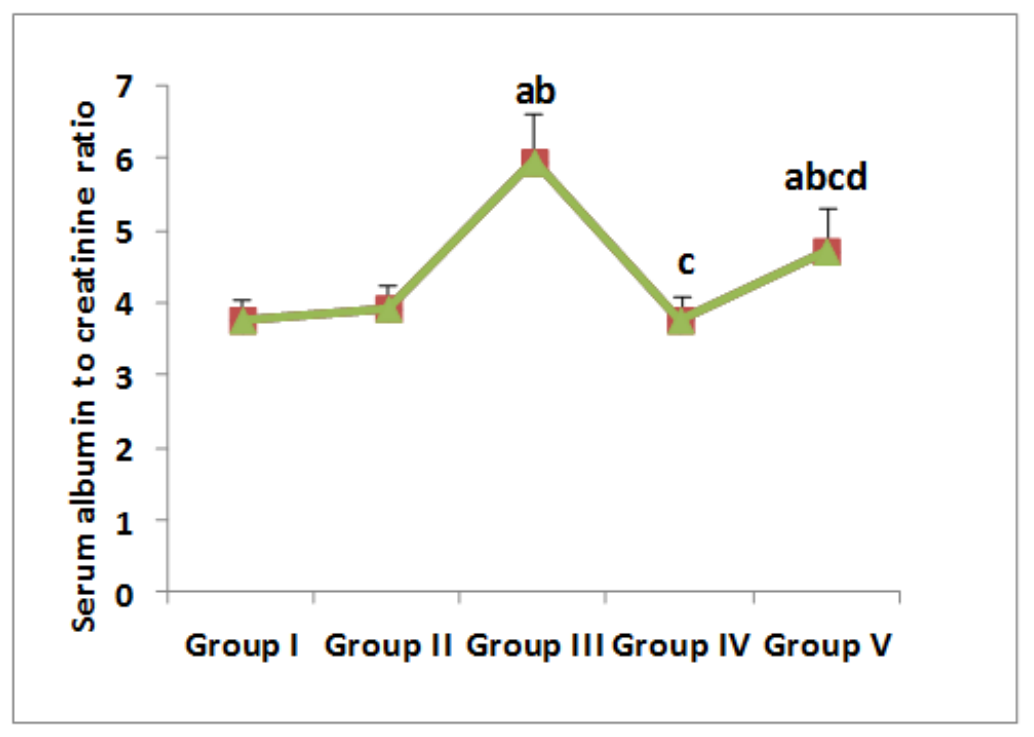

Figure 1 Serum albumin to creatinine ratio 
Values are provided as Mean \pm SD for six animals of each group. a, b, c, d indicate significant differences from the normal control, astaxanthin control; calculi induced group and the astaxanthin treated group respectively. $\mathrm{p}<0.05$ was considered significant.

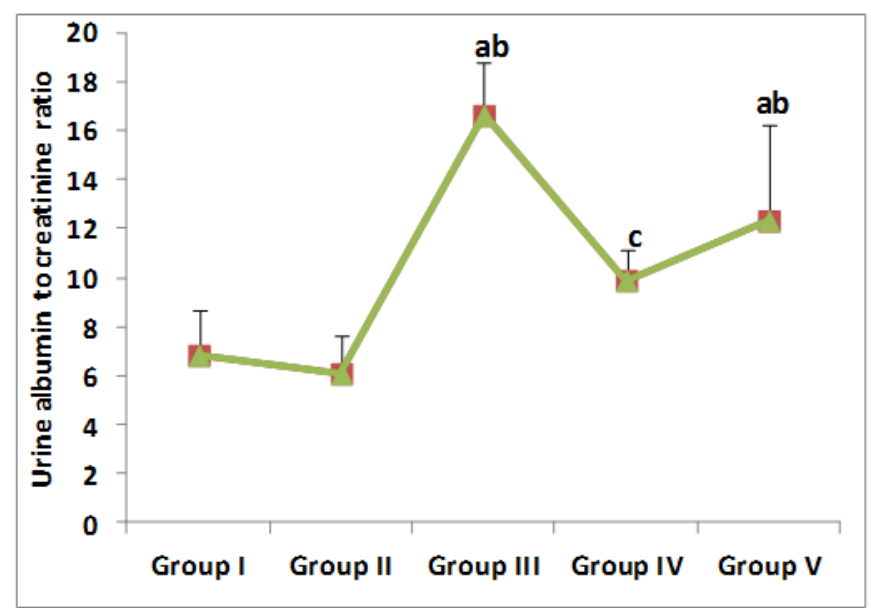

Figure 2 Urine albumin to creatinine ratio

Values are provided as Mean \pm SD for six animals of each group. a, b, c, d indicate significant differences from the normal control, astaxanthin control; calculi induced group and the astaxanthin treated group respectively. $\mathrm{p}<0.05$ was considered significant.

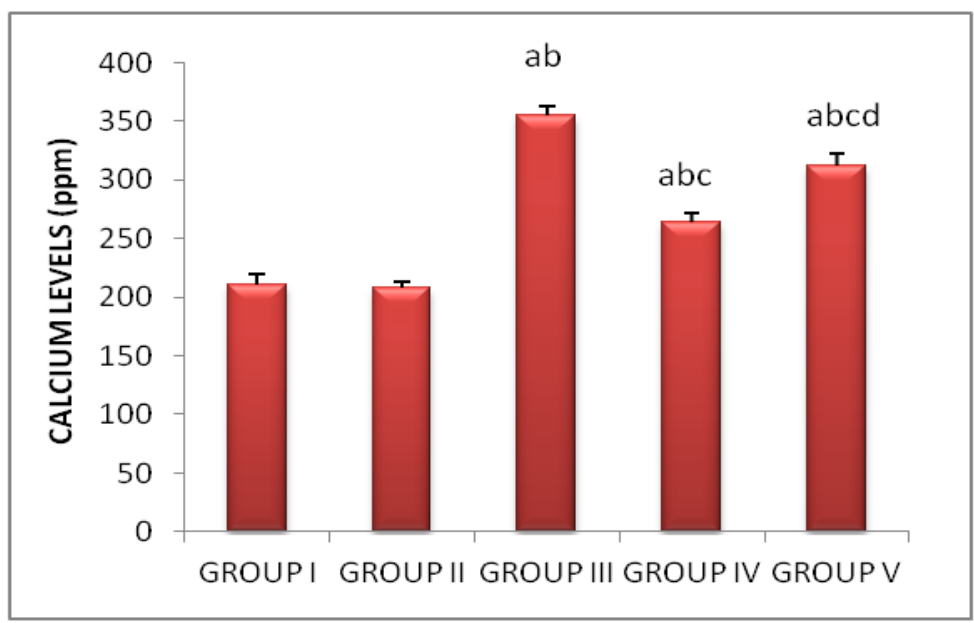

Figure 3 Calcium content in the kidney tissue (ppm)

Values are provided as Mean \pm SD for six animals of each group. a, b ,c, d indicate significant differences from the normal control, astaxanthin control; calculi induced group and the astaxanthin treated group respectively. $\mathrm{p}<0.05$ was considered significant.

Table 1 The body weights of the animals on the first day and at the end of the experimental period

\begin{tabular}{|c|c|c|}
\hline Experimental Groups & Initial body weight $(\mathrm{g})$ & Final body weight $(\mathrm{g})$ \\
\hline Group I & $253 \pm 8.89$ & $261.5 \pm 8.07$ \\
\hline Group II & $245.17 \pm 5.15$ & $255 \pm 15.75$. \\
\hline Group III & $238.16 \pm 11.6$ & $223.67 \pm 4.18^{\text {ab }}$ \\
\hline Group IV & $245.62 \pm 6.03$ & $252.5 \pm 6.3^{\mathrm{c}}(76.21 \%)$ \\
\hline Group V & $240.02 \pm 2.03$ & $248.5 \pm 6.76^{\mathrm{c}}(65.63 \%)$ \\
\hline
\end{tabular}

Values are provided as Mean \pm SD for six animals of each group. a,b,c,d indicate significant differences from the normal control, astaxanthin control; calculi induced group and the astaxanthin treated group respectively. $\mathrm{p}<0.05$ was considered significant. 
Table 2 Relative kidney weight percentage

\begin{tabular}{|c|c|c|}
\hline Experimental Groups & Absolute kidney weight $(\mathrm{g})$ & Relative kidney weight (\%) \\
\hline Group I & $1.418 \pm .102$ & 0.54 \\
\hline Group II & $1.425 \pm 0.105$ & 0.55 \\
\hline Group III & $1.6933 \pm 0.047^{\mathrm{ab}}$ & $0.75^{\mathrm{ab}}$ \\
\hline Group IV & $1.506 \pm 0.042^{\mathrm{c}}$ & $0.59^{\mathrm{ac}}$ \\
\hline Group V & $1.56 \pm 0.058^{\mathrm{c}}$ & $0.64^{\text {abcd }}$ \\
\hline
\end{tabular}

Values are provided as Mean \pm SD for six animals of each group.

$\mathrm{a}, \mathrm{b}, \mathrm{c}, \mathrm{d}$ indicate significant differences from the normal control, astaxanthin control; calculi induced group and the astaxanthin treated group respectively. $\mathrm{p}<0.05$ was considered significant.

Table 3 Serum Marker enzymes

\begin{tabular}{|l|l|l|l|}
\hline Experimental Groups & AST(U/L) & ALT(U/L) & ALP(U/L) \\
\hline Group I & $18.86 \pm 2.21$ & $76.03 \pm 1.37$ & $70.95 \pm 1.68$ \\
\hline Group II & $20.17 \pm 1.47$ & $79.67 \pm 1.53$ & $66.03 \pm 1.75$ \\
\hline Group III & $27.37 \pm 0.96^{\text {ab }}$ & $103.012 \pm 2.84^{\text {ab }}$ & $149.37 \pm 4.24^{\text {ab }}$ \\
\hline Group IV & $19.85 \pm 1.17^{\mathrm{c}}$ & $98.65 \pm 2.31^{\text {abc }}$ & $92.37 \pm 0.87^{\text {abc }}$ \\
\hline Group V & $23.3 \pm 0.87^{\text {abcd }}$ & $95.69 \pm 1.30^{\text {abc }}$ & $115.29 \pm 5.79^{\text {abcd }}$ \\
\hline
\end{tabular}

Values are provided as Mean \pm SD for six animals of each group.

a,b,c,d indicate significant differences from the normal control, astaxanthin control; calculi induced group and the astaxanthin treated group respectively. $\mathrm{p}<0.05$ was considered significant.

Table 4 Effect of astaxanthin on lipid peroxidation and liver antioxidants

\begin{tabular}{|l|l|l|l|l|l|}
\hline $\begin{array}{l}\text { Experiment } \\
\text { al Groups }\end{array}$ & $\begin{array}{l}\text { TBARS } \\
(\mathrm{nmol} \text { of TBARS } \\
\text { formed/min/mg } \\
\text { protein) }\end{array}$ & $\begin{array}{l}\text { SOD } \\
(\mathrm{U} / \mathrm{mg} \text { protein) }\end{array}$ & $\begin{array}{l}\text { CAT } \\
(\mathrm{nkat} / \mathrm{mg} \\
\text { protein) }\end{array}$ & $\begin{array}{l}\text { GPx } \\
(\mu \mathrm{g} \text { of GSH } \\
\text { consumed/min/mg } \\
\text { protein })\end{array}$ & $\begin{array}{l}\text { GST( } \mu \text { mol of CDNB } \\
\text { formed/min/mg } \\
\text { protein) }\end{array}$ \\
\hline Group I & $4.91 \pm 0.037$ & $7.36 \pm 0.29$ & $52.61 \pm 0.88$ & $3.87 \pm 0.51$ & $24.93 \pm 2.03$ \\
\hline Group II & $4.53 \pm .052$ & $8.59 \pm 0.39^{\mathrm{a}}$ & $61.769 \pm 1.73^{\mathrm{a}}$ & $4.711 \pm 0.10$ & $25.49 \pm 2.28$ \\
\hline Group III & $7.62 \pm 0.17^{\mathrm{ab}}$ & $3.76 \pm 0.43^{\mathrm{ab}}$ & $30.06 \pm 1.16^{\mathrm{abc}}$ & $2.79 \pm 0.13^{\mathrm{ab}}$ & $34.53 \pm 3.14^{\mathrm{ab}}$ \\
\hline Group IV & $4.02 \pm 0.03^{\mathrm{c}}$ & $6.72 \pm 0.12^{\mathrm{c}}$ & $50.09 \pm 3.14^{\mathrm{bcd}}$ & $4.22 \pm 0.07^{\mathrm{bcd}}$ & $23.7 \pm 2.04$ \\
\hline Group V & $6.02 \pm 0.2^{\text {abd }}$ & $4.09 \pm 0.23$ & $32 \pm 0.22$ & $3.09 \pm 0.09^{\mathrm{abd}}$ & $30 \pm 0.12^{\mathrm{abd}}$ \\
\hline
\end{tabular}

Values are provided as Mean \pm SD for six animals of each group.

a,b,c,d indicate significant differences from the normal control, astaxanthin control; calculi induced group and the astaxanthin treated group respectively. $\mathrm{p}<0.05$ was considered significant.

\section{Discussion}

Coadministration of ethylene glycol and Vitamin $\mathrm{D}_{3}$ is used as an accelerated model to produce COM stones in the kidney. Ethylene glycol is metabolised to glycolate and glyoxylate which cause tissue destruction particularly due to COM deposition in the renal tissue. The oxalic acid formed by the metabolism combines with calcium to form calcium oxalate which gets deposited in the kidney leading to renal failure [15].

Organ to body weight ratio is a marker of cell constriction and inflammation [16]. In the present study astaxanthin is found to exert renal protection against the calculi induced damages as is depicted by the reduction in the kidney weight to body weight ratio and higher renoprotective index when compared to the citrate administered group.

Increase in serum enzymes is roughly proportional to the extent of tissue damage [17]. The common enzymes employed as indicators of hepatocellular damage are AST, ALT and ALP. We have also assessed these diagnostic markers to evaluate the activity of astaxanthin in liver. The result of our experiment also shows an increase in all the three enzyme activities in the calculi induced group. This could be possibly due to leakage of cytoplasmic enzymes into circulation due to inflammation of liver cells. However in the treated groups, the AST and ALT activities show a return to the normal levels with the astaxanthin treatment reducing the enzyme elevations more effectively than the citrate treatment. This shows the effect of astaxanthin in preserving the liver cell integrity. There is also a reduction in the ALP levels in both the treated groups with astaxanthin group exhibiting a significant reduction than the citrate treated group. Previous studies showing a similar effect attributes this restorative effect of astaxanthin to its superior antioxidant activity and free radical scavenging effect. The ALP activity restoration is an indication of stabilization of plasma membrane as well as repair of 
hepatic tissue damage. This can be due to a functional improvement of hepatocytes which may be caused by an accelerated regeneration of parenchyma cells [18].

SOD catalyses the scavenging of superoxide radicals and renders cytoprotection against free radical damage. CAT and GPx are involved in elimination of Hydrogen peroxide. GST helps in conjugating molecules to GSH and detoxifies cellular environment. The treatment with astaxanthin is effective in reducing the lipid peroxidation and enhancing the activities of SOD, CAT, GPx and GST in the liver tissue. The unique structure of astaxanthin with a keto and hydroxyl group at each end must be associated with its potent antiperoxidation activity. This is believed to rigidify the biomembranes thus limiting the passage of lipoperoxidation promoters across the cell membranes. [19] Astaxanthin acts as a strong antioxidant by donating the electrons and reacting with free radicals to convert them to more stable product and terminates free radical chain reactions in a wide variety of living organisms [20].

The ratio of concentration of albumin to creatinine in urine is used as index of urine albumin concentration used to diagnose early stages of renal damage [21]. An increase in serum ACR indicates microalbuminuria and slight kidney dysfunction [22].The results of our study show a significant elevation in the ACR of the calculi induced group when compared to the controls. A reduction in the astaxanthin treated groups therefore indicates the ability of astaxanthin to increase the glomerular filteration rate. The administration of astaxanthin is also found to decrease the calcium accumulation in the renal tissue as seen from the results of flame photometry. Thus it can be summarised that astaxanthin treatment is effective in ameliorating the hepatic and renal damage caused in experimentally induced nephrolithiasis.

\section{Conclusion}

Treatment with astaxanthin at $25 \mathrm{mg} / \mathrm{kg}$ body weight causes recovery of bodyweight, improved the liver function markers and increased the glomerular filteration rate. The lipid peroxidation of the hepatic tissues decreased and the antioxidant enzyme levels are restored to normal levels. Hence astaxanthin can be used effectively as a nutraceutical against nephrolithiasis. Furthur studies are needed to confirm these observations.

\section{References}

[1] JR Asplin. Hyperoxaluric calcium nephrolithiasis. Endocrinology and Metabolism Clinics of North America 31(4), 2002, 927949.

[2] S Jafar, L Mehri, B Hadi and M Jamshid. The antiurolithiasic and hepatocurative activities of aqueous extracts of Petroselinum sativum on ethylene glycol-induced kidney calculi in rats. Science Research Essays 7, 2012, 1577-1583.

[3] SA Bailey, RH Zidell and RW Perry. Relationship between organ weight and body/brain weight in the rat: what is the best analytical endpoint? Toxicological Patholology 32(4), 2004, 448-466.

[4] Singh A, Bhat TK, Sharma OP. Clinical Biochemistry of Hepatotoxicity. Journal of Clinical Toxicology 2011, S4:001.

[5] G Hussein, U Sankawa, H Goto, K Matsumoto and H Watanabe. Astaxanthin, a carotenoid with potential in human health and nutrition. Journal of Natural Products , 69(3), 2006, 443-449.

[6] Shimidzu, Gogo and Miki. Carotenoids as Singlet Oxygen Quenchers in Marine Organisms. Fisheries Science 62(1), 1995, 134137.

[7] M Guerin, ME Huntley and M Olaizola. Haematococcus astaxanthin:applications for human health and nutrition, Trends in Biotechnology, 21(5), 2003, 210-216.

[8] S Saha and RJ Verma. Bergenia ciliata extract prevents ethylene glycol induced histopathological changes in the kidney. Acta Poloniae Pharmaceutica and Drug Research, 68 (5), 2011, 711-715.

[9] JA Beuge and SD Aust. The thiobarbituric acid assay. Methods in Enzymology 52, 1978, 306-307.

[10] P Kakkar, B Das and PN Viswanathan. A modified spectrophotometric assay of superoxide dismutase. Indian Journal of Biochemistry and Biophysics 21, 1984, 130-132.

[11] H Aebi. Catalase: In Bergmeyer, H.U (Ed.), Methods of Enzymatic Analysis, Vol. II. 2 (Academic Press, New York, 1974$) 673-684$.

[12] JT Rotruck, AL Pope and HE Ganther. Selenium: biochemical role as a component of glutathione peroxidase purification and assay. Science, 179, 1973, 588-590.

[13] WH Habig, MJ Pabst and WB Jakoby. Glutathione S-transferase, the first enzymatic step in mercapturic acid formation. Journal of Biological Chemistry 249, 1974, 7130-7139.

[14] OH Lowry, NJ Rosenbourgh, AL Farr and RJ Randall. Protein measurement with folin phenol reagent. Journal of Biological Chemistry, 193, 1951, 265-275.

[15] Y Li and KE McMartin. Strain differences in urinary factors that promote calcium oxalate crystal formation in the kidneys of ethylene glycol-treated rats. American Journal of Physiology-Renal Physiology, 296, 2009, 1080-1087.

[16] KL Moore and AF Dalley. Structure of the penis. In: Clinical Oriented Anatomy. 4 (Philadelphia: Lippincot Williams and Williams, 1999) 287-299.

[17] A Gaw, RA Cowman, DS O'Reilly and J Shepherd. Clinical Biochemistry: An Illustrated Color Text. Clinical Biochemistry (Elsevier, New York. 1995) 49.

[18] J Rajangam and AJN Christina. Evaluation of Hepatoprotective and antioxidant potential of methanolic extract of Polyalthiya longifolia fruits: An in-vitro and in-vivo approach. Journal of Applied Pharmaceutical Science, 3(2), 2013, 69-76.

[19] A Wisniewska and WK Subczynski. Effects of polar carotenoids on the shape of the hydrophobic barrier of phospholipids bilayers. Biochimica et Biophysica Acta, 1368, 1998, 235-246.

[20] JP Yuan, J Peng, KYin, JH Wang. Potential health-promoting effects of astaxanthin: A high-value carotenoid mostly from microalgae. Molecular Nutrition \& Food Research, 2011, 55, 150-165.

[21] Urine albumin to creatinine ratio in evaluating patients with diabetes for kidney disease. NIH Publication No. 08-6286, 2008.

[22] S Khan 2010. Albumin creatinine ratio. http://www.buzzle.com/articles/albumin-creatinine-ratio.html. 